University of New Hampshire

University of New Hampshire Scholars' Repository

Space Science Center

Institute for the Study of Earth, Oceans, and

Space (EOS)

1994

\title{
MeV emission from the black-hole candidate GRO J0422+32 measured with COMPTEL
}

\author{
R VanDijk \\ ESTEC \\ H Bloemen \\ Space Research Organization of the Netherlands \\ W Hermsen \\ Space Research Organization of the Netherlands (SRON) \\ W Collmar \\ Max-Planck-Institut für extraterrestriche Physik \\ R Diehl \\ Max-Planck-Institut für extraterrestriche
}

See next page for additional authors

Follow this and additional works at: https://scholars.unh.edu/ssc

Part of the Astrophysics and Astronomy Commons

\section{Recommended Citation \\ MeV emission from the black-hole candidate GRO J0422+32 measured with COMPTEL van Dijk, R. and Bloemen, $\mathrm{H}$. and Hermsen, W. and Collmar, W. and Diehl, R. and Greiner, J. and Lichti, G. G. and Schönfelder, V. and Strong, A. and Bennett, K. and Hanlon, L. and Winkler, C. and McConnell, M. and Ryan, J., AIP Conference Proceedings, 304, 197-201 (1994), DOI:http://dx.doi.org/10.1063/1.45619}

This Conference Proceeding is brought to you for free and open access by the Institute for the Study of Earth, Oceans, and Space (EOS) at University of New Hampshire Scholars' Repository. It has been accepted for inclusion in Space Science Center by an authorized administrator of University of New Hampshire Scholars' Repository. For more information, please contact Scholarly.Communication@unh.edu. 


\section{Authors}

R VanDijk, H Bloemen, W Hermsen, W Collmar, R Diehl, J Greiner, G G. Lichti, V Schonfelder, A W. Strong, K Bennett, L O. Hanlon, C Winkler, Mark L. McConnell, and James M. Ryan 


\section{AIP $\mid$ proceedings}

\section{MeV emission from the blackhole candidate GRO J0422+32 measured with COMPTEL}

R. van Dijk, H. Bloemen, W. Hermsen, W. Collmar, R. Diehl, J. Greiner, G. G. Lichti , V. Schönfelder, A. Strong, K. Bennett, L. Hanlon, C. Winkler, M. McConnell, and J. Ryan

Citation: AIP Conference Proceedings 304, 197 (1994); doi: 10.1063/1.45619

View online: http://dx.doi.org/10.1063/1.45619

View Table of Contents:

http://scitation.aip.org/content/aip/proceeding/aipcp/304?ver=pdfcov

Published by the AIP Publishing

\section{Articles you may be interested in}

A transient $\mathrm{MeV}$ range gammaray source observed by HEAO-1

AIP Conf. Proc. 304, 255 (1994); 10.1063/1.45628

BATSE observations of transient hard Xray sources AIP Conf. Proc. 304, 210 (1994); 10.1063/1.45621

Timing noise properties of GRO J0422+32

AIP Conf. Proc. 304, 192 (1994); 10.1063/1.45618

COMPTEL detection of the variable radio source GT $0236+610$ AIP Conf. Proc. 304, 324 (1994); 10.1063/1.45581

Xray and optical observations of GRO J0422+32

AIP Conf. Proc. 304, 319 (1994); 10.1063/1.45580 


\title{
MeV EMISSION FROM THE BLACK-HOLE CANDIDATE GRO J0422+32 MEASURED WITH COMPTEL
}

\author{
R. van Dijk ${ }^{1}$, H. Bloemen, W. Hermsen \\ SRON-Leiden, P.O. Box 9504, NL-2300 RA Leiden, The Netherlands
}

W. Collmar, R. Diehl, J. Greiner, G.G. Lichti, V. Schönfelder, A. Strong

Max-Planck Institute für Extraterrestrische Physik, P.O. Box 1603, 85740 Garching, F.R.G.

K. Bennett, L. Hanlon, C. Winkler

Astrophysics Division, ESTEC, NL-2200 AG Noordwijk, The Netherlands

M. McConnell, J. Ryan

Space Science Center, Univ. of New Hampshire, Durham NH 03824, U.S.A.

\begin{abstract}
On Aug. 5, 1992 BATSE discovered the bright X-ray transient GRO J0422+32, also known as Nova Per 1992. The COMPTEL instrument $(0.75-30 \mathrm{MeV})$ aboard CGRO observed this black-hole candidate twice. During the first observation, which started when the X-ray flux was at its maximum, GRO J0422+32 was detected up to $2 \mathrm{MeV}$. The fluxes in the 0.75-1 and 1-2 MeV range were significantly higher than expected based on an extrapolation of the contemporaneous OSSE data. The COMPTEL spectral points can be interpreted as evidence for a Wien-type spectral component with temperature $k T \approx 300 \mathrm{keV}$. During the second obervation, 3 weeks after the first, GRO J0422+32 was detected only up to $1 \mathrm{MeV}$.
\end{abstract}

\section{INTRODUCTION}

GRO J0422+32 was discovered on Aug. 5, 1992 with the BATSE instrument aboard the Compton Gamma-Ray Observatory (CGRO). The intensity in the $20-300 \mathrm{keV}$ range on August 8 was $\sim 0.2 \times$ Crab (Paciesas et al. 1992) and within a few days increased to $\sim 3 \times$ Crab, remaining at that level for another 3 days (Harmon et al. 1992a). After the initial outburst, GRO J0422+32 decreased exponentially with a decay time of $\sim 41$ days (Vikhlinin et al. 1992). In the first half of December 1992, a secondary maximum occurred in hard X-rays (Harmon et al. 1992b) and probably also in the optical (Chevalier \& Dovaisky 1993). The source showed strong variability on all time scales in X-rays and was seen up to $600 \mathrm{keV}$ by the OSSE instrument aboard CGRO. The early OSSE data are well represented by a two temperature Sunyaev-Titarchuk model with temperatures $30 \mathrm{keV}$ and $60 \mathrm{keV}$ and optical depths of 6 and 3 respectively (Cameron et al. 1992).

The COMPTEL observations presented in this paper show significant emission up to 2 $\mathrm{MeV}$. The high-energy emission of GRO J0422+32 and the similarity of the outburst characteristics to black-hole candidates such as A0620-00 and GS2023+338, suggest that GRO J0422+32 is also a (weak) black-hole candidate (Liang 1992). Kato et al. (1993), assuming that the observed optical modulation is caused by superhumps, derive a mass for the compact object in the range $2.9 \mathrm{M}_{\odot}-6.2 \mathrm{M}_{\odot}$. Future photometric and spectroscopic observations may confirm its black-hole nature by determining the mass function.

\footnotetext{
${ }^{1}$ Also Astronomical Institute, University of Amsterdam, Kruislaan 403, NL-1098 SJ Amsterdam, The Netherlands
} 


\section{THE INSTRUMENT}

COMPTEL is one of four instruments aboard CGRO which was launched in April 1991. It is operating in the $0.75-30 \mathrm{MeV}$ energy range, thereby forming a link between the hard $\mathrm{X}$ rays/low-energy $\gamma$-rays (measured by OSSE and BATSE aboard CGRO) and the $>30 \mathrm{MeV} \gamma$ rays (measured by EGRET, also aboard CGRO). COMPTEL is a wide field-of-view imaging instrument ( 1 steradian) with a positional accuracy of about $0.3^{\circ}$ for a strong source and an energy resolution of $5 \%$ FWHM at $1 \mathrm{MeV}$. The detection mechanism is based on Compton scattering, the dominant photon-matter interaction mechanism in this energy range. For a complete description of the instrument characteristics the reader is referred to Schönfelder et al. (1993).

\section{OBSERVATIONS AND RESULTS}

COMPTEL observed GRO J0422+32 on two occasions in 1992 (Table 1). The first observation (36), lasting 9 days, covers the plateau-like maximum of the $\mathrm{X}$-ray light-curve just after GRO J0422+32 was discovered (see Harmon et al. 1992). It consists of two parts, Obs. 36.0 and 36.5 , which differ only by a rotation of the spacecraft around the pointing (in Obs. 36.5 the solar aspect angle had been optimised). In the second observation (39.0), lasting 16 days, the average $\mathrm{X}$-ray flux had decreased to $\sim 70 \%$ of its maximum value. For the analysis presented here, only Obs. 36.5 and 39.0. have been considered.

Table I. List of observations (1992). The 4 columns show: 1) the observation ID in GRO notation, 2) the angular distance $\zeta$ to the pointing direction, 3) the start date and time and 4) the end date and time.

\begin{tabular}{ccll}
\hline Obs & $\begin{array}{c}\zeta \\
{\left[{ }^{\circ}\right]}\end{array}$ & Start & End \\
\hline 36.0 & 3.91 & Aug. 11, 01:59 UT & Aug 12, 18:22 UT \\
36.5 & 3.32 & Aug. 12, 19:00 UT & Aug 20, 15:28 UT \\
39.0 & 2.99 & Sep. 01, 05:40 UT & Sep 17, 15:17 UT \\
\hline
\end{tabular}

The COMPTEL software system supports two methods of creating sky maps: a maximumlikelihood ratio method (ML) and a maximum-entropy method (ME). Detailed descriptions of the two methods can be found elsewhere (de Boer et al. 1992; Strong et al. 1992; Bloemen et al. 1993; Schönfelder et al. 1993). The application of both the ML and the ME method requires the assumption of an input spectrum. For the analysis of GRO J0422+32, Wien input spectra were used (with $F \propto x^{2} \exp (-x), x \equiv h \nu / k T$ ), because this type of spectrum is expected at COMPTEL energies when the photons are produced through inverse-Compton scattering (Cameron et al. 1992; Sunyaev \& Titarchuk 1980). Additionally, power-law input spectra were also considered.

Using the ML method, we have detected GRO J0422+32 in Obs. 36.5 in the $0.75-2 \mathrm{MeV}$ energy range with a significance of $\sim 3.5 \sigma$. In Obs. 39.0, which started 3 weeks after the initial outburst, we only obtain a flux point $(\sim 2.2 \sigma)$ in the $0.75-1 \mathrm{MeV}$ range. Fig. 1 shows the sky map for the $0.75-2 \mathrm{MeV}$ energy range in Obs. 36.5 obtained with the ML method. It shows the $95 \%$ and $99 \%$ location contours of GRO J0422+32 and the $95 \%$ and $99 \%$ location contours of Crab in a field which is empty except for these two sources. Note that the quasar PKS 0528+134 reported by Collmar et al. (1992), located only 5 degrees from the Crab, is not detected by COMPTEL at these low energies. GRO J0422+32 lies roughly at the $80 \%$ location contour. 
Table II. $0.75-1$ and $1-2 \mathrm{MeV}$ fluxes for Obs. 36.5 and 39.0 . The $k T$ values refer to the temperatures of the Wien input spectra.

\begin{tabular}{lllcccc}
\hline Obs & $\begin{array}{l}\mathrm{E} \\
\end{array}$ & & \multicolumn{5}{c}{ Flux } & & \\
& {$[\mathrm{MeV}]$} & & \multicolumn{4}{c}{$\left[\mathrm{cm}^{-2} \mathrm{~s}^{-1} \mathrm{MeV}^{-1}\right]$} \\
& & $k T=80 \mathrm{keV}$ & $k T=100 \mathrm{keV}$ & $k T=150 \mathrm{keV}$ & $k T=200 \mathrm{keV}$ & $k T=300 \mathrm{keV}$ \\
\hline 36.5 & $0.75-1$ & $(6.3 \pm 2.7) 10^{-4}$ & $(6.1 \pm 2.7) 10^{-4}$ & $(5.8 \pm 2.5) 10^{-4}$ & $(5.7 \pm 2.5) 10^{-4}$ & $(5.6 \pm 2.4) 10^{-4}$ \\
& $1-2$ & $(1.9 \pm 0.6) 10^{-4}$ & $(1.9 \pm 0.6) 10^{-4}$ & $(1.8 \pm 0.6) 10^{-4}$ & $(1.7 \pm 0.6) 10^{-4}$ & $(1.6 \pm 0.6) 10^{-4}$ \\
39.0 & $0.75-1$ & $(4.0 \pm 2.1) 10^{-4}$ & $(4.2 \pm 2.1) 10^{-4}$ & $(3.9 \pm 1.9) 10^{-4}$ & $(3.8 \pm 1.9) 10^{-4}$ & $(3.9 \pm 1.9) 10^{-4}$ \\
\hline
\end{tabular}

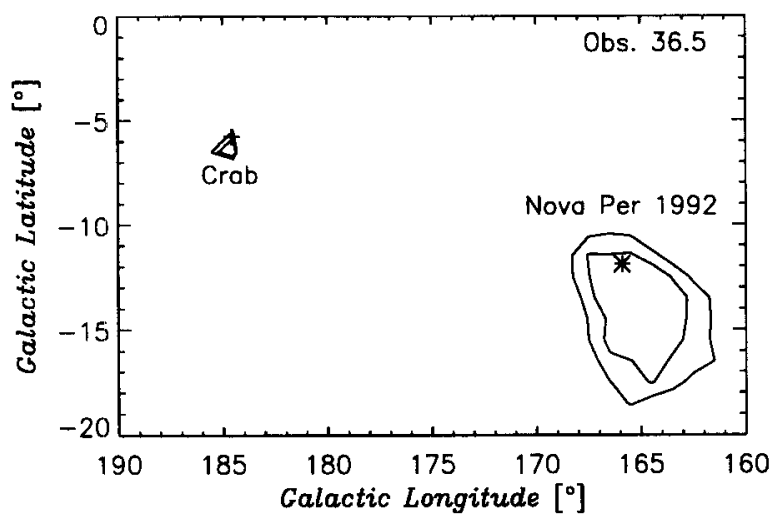

Fig 1. Skymap for the $0.75-2 \mathrm{MeV}$ range for Obs. 36.5 . The contours are the $95 \%$ and $99 \%$ error location contours.

Table 2 lists the fluxes and errors per observation period for the $0.75-1$ and $1-2 \mathrm{MeV}$ energy ranges. The analysis was performed assuming Wien input spectra and repeated for several values of the temperature $k T$. It is evident that the derived flux values and therefore also the ratios of the $0.75-1$ and $1-2 \mathrm{MeV}$ fluxes, are only slightly dependent on the assumed temperature. Comparing these ratios with the ratios expected for Wien spectra, we can constrain the temperature of the Wien component in Obs. 36.5 to $k T=300_{-100}^{+200} \mathrm{keV}$, with a firm lower limit of $150 \mathrm{keV}$. In Obs. 39.0 the $0.75-1 \mathrm{MeV}$ flux is about $25 \%$ lower compared to that of Obs. 36.5. Such a variation is consistent with the $\mathrm{X}$-ray light-curve as observed by BATSE (Harmon et al. 1992), but due to the large statistical uncertainties it is also consistent with no time variation at all.

When a power-law input spectrum is assumed in the $0.75-1$ and $1-2 \mathrm{MeV}$ ranges, a similar analysis constrains the photon index $\alpha$ between 1 and 3 . The fluxes and the upper limits in the energy ranges above $2 \mathrm{MeV}$ do not rule out a power-law spectrum.

Fig. 2 shows the COMPTEL spectral points and upper limits (for a Wien input spectrum with $k T=300 \mathrm{keV}$ ) from Obs. 36.5, together with the two-component Sunyaev-Titarchuk model fit to the contemperaneous OSSE data $(\tau=6, k T=30 \mathrm{keV}$ and $\tau=3, k T=60$ $\mathrm{keV}$ respectively; Cameron et al. 1992). The COMPTEL flux values are clearly higher than expected from an extrapolation of the fit. For comparison a Wien spectrum with $k T=300$ $\mathrm{keV}$ is also shown (dashed-dotted line). 


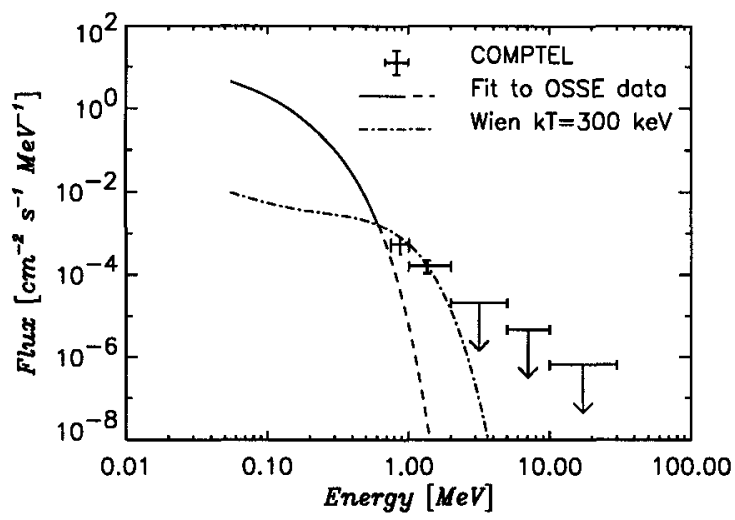

Fig 2. The COMPTEL fluxes and upper limits for Obs. 36.5 together with the extrapolated two-component Sunyaev-Titarchuk model fit to the contemporaneous OSSE data (Cameron et al. 1992). The fluxes were derived assuming a Wien spectrum $(k T=300 \mathrm{keV})$ as input spectrum.

\section{DISCUSSION}

The X-and $\gamma$-ray spectra of black-hole candidates are now known to consist of several variable components. Below $100 \mathrm{keV}$ the spectra can generally be approximated by a hard power-law like component with an ultra-soft excess. Both are observed to be highly variable and may disappear completely (e.g. Ebisawa 1991; Grebenev et al. 1993). Between $\sim 1-20$ $\mathrm{keV}$, the spectrum is more complex and may show an iron- $\mathrm{K}$ emission line and a reflection component (Ebisawa 1991; Done et al. 1992; Haardt et al. 1993).

Detections of transient features at $1 \mathrm{MeV}$ and above in the spectra of the black-hole candidate Cyg X-1 have been reported on several occasions. McConnell et al. (1989) reported a $2.9 \sigma$ detection of Cyg X-1 in the $2-9.3 \mathrm{MeV}$ energy range during observations in 1984 . Three observations made with the HEAO A3 instrument in 1979 and 1980, showed Cyg X-1 in three distinct spectral states, which were called $\gamma_{1}, \gamma_{2}$ and $\gamma_{3}$. In the $\gamma_{1}$ state, which is defined by a low $45-140 \mathrm{keV}$ flux, the spectrum showed a broad Gaussian feature centered at $\sim 965 \mathrm{keV}$ with a FWHM of $\sim 1173 \mathrm{keV}$ (Ling et al. 1987). Observations with COMPTEL did not reveal excess $\mathrm{MeV}$ emission above the extrapolated fits to the OSSE data, indicating that any existing $\mathrm{MeV}$ emission must be variable (McConnell et al. 1993).

Liang \& Dermer (1988) and Liang (1990) modelled the $\gamma_{1}$ spectrum of Cyg X-1 by incorporating a hot, pair dominated, cloud $(k T \sim 400 \mathrm{keV})$ in the inner disk region. For this cloud to become visible at $\mathrm{MeV}$ energies, a transition zone was introduced between the inner and outer disk regions, which prevents the soft photons originating in the cool outer parts from cooling the hot plasma cloud. Melia \& Misra (1993) proposed a similar model in which the high-energy photons are produced in the two-temperature inner region of the disk by up-scattering of cool disk photons and internal bremsstrahlung photons. In this model, a shield is naturally established when the viscosity parameter $\alpha$ decreases a factor 2 from its value in the $\gamma_{2}$ state.

The COMPTEL flux points for GRO J0422+32 are significantly above the extrapolation of the contemporaneous OSSE data. A qualitative comparison of the $\gamma$-ray spectrum measured by OSSE and COMPTEL with the model of Melia \& Misra (1993) for the $\gamma_{2}$ state of Cyg $\mathrm{X}-1$ shows good agreement. 
A question which arises is whether the shape of the spectrum is time variable. We detect GRO J0422+32 at $\sim 3 \sigma$ in the $1-2 \mathrm{MeV}$ energy range in Obs. 36.5, but there is only a upper limit of $8.2 \times 10^{-5} \mathrm{~cm}^{-2} \mathrm{~s}^{-1} \mathrm{MeV}^{-1}(2 \sigma)$ in Obs. 39.0. Taking the values from Table 1 and assuming no time variations of the shape of the spectrum, we would expect to measure a 1-2 $\mathrm{MeV}$ flux of $1.1 \times 10^{-4} \mathrm{~cm}^{-2} \mathrm{~s}^{-1} \mathrm{MeV}^{-1}$ in Obs. 39.0. Although the upper limit for the 1-2 $\mathrm{MeV}$ energy range in Obs. 39.0 might suggest time variation, the errors on the fluxes in Table 1 are too large to support this conclusion.

In conclusion, the COMPTEL instrument aboard CGRO detected GRO J0422+32 up to $2 \mathrm{MeV}$ in Obs. 36.5 and up to $1 \mathrm{MeV}$ in Obs. 39.0. The $0.75-1$ and $1-2 \mathrm{MeV}$ flux points for Obs. 36.5 are significantly above the extrapolation of the fit to the contemporaneous OSSE data. An analysis of the combination of the OSSE and COMPTEL data is required to test the various existing models. It is evident that sensitive future studies in the region around 1 $\mathrm{MeV}$ will provide valuable information for our understanding of sources like GRO J0422+32. A detailed description of the present findings is given by van Dijk et al. (1994).

\section{REFERENCES}

Bloemen H. et al., 1993, to appear in ApJ Suppl. (proceedings INTEGRAL workshop)

Cameron R.A. et al., 1992, IAU Circ 5587

Chevalier C., Ilovaisky S.A., 1993, IAU Circ 5692

Collmar W. et al., 1992, in: Proceedings of the COMPTON Gamma-Ray Observatory, St. Louis, AIP 280, p483

de Boer H. et al., 1992, in: Data Analysis in Astronomy IV, eds. V. Di Gesù (New York: Plenum Press), Vol 59, p241

Done C. et al., 1992, ApJ 395, 275

Ebisawa K. et al., 1991, PhD Thesis, University of Tokyo, Japan

Grebenev S. et al., 1993, A\&AS 97, 281

Haardt F. et al., 1993, ApJ 411, L95

Harmon B.A. et al., 1992a, IAU Circ. 5584

Harmon B.A. et al., 1992b, IAU Circ. 5685

Harmon B.A. et al., 1992, in: Proceedings of the COMPTON Gamma-Ray Observatory, St. Louis, AIP 280, p314

Kato T., Mineshige S., Hirata R., 1993, IAU Circ 5704

Liang E.P., Dermer C.D., 1988, ApJ 325, L39

Liang E.P., 1990, A\&A 227, 447

Liang E.P., 1992, in: Proceedings of the COMPTON Gamma-Ray Observatory, St. Louis, AIP 280, p396

Ling J.C. et al., 1987, ApJ 321, L117

McConnell M.L. et al., 1989, ApJ 343, 317

McConnell M.L. et al., 1993, ApJ, accepted

Melia F., Misra R., 1993, ApJ 411, 797

Paciesas W.S., Briggs M.S., Harmon B.A., Wilson R.B., Finger M.H., 1992, IAU Circ. 5580

Schönfelder et al., 1993, ApJS 86, 657

Strong A.W. et al., 1992, in: Data Analysis in Astronomy IV, eds. V. Di Gesù (New York: Plenum Press), Vol 59, p251

Sunyaev R.A., Titarchuk L.G., 1980, A\&A 86, 121

van Dijk R. et al., 1994, A\&A, in preparation

Vikhlinin A. et al., 1992, IAU Circ 5608 